OPEN ACCESS

Edited by:

Hongyan Wang,

Duke-NUS Medical School,

Singapore

Reviewed by:

S. Sean Millard,

The University of Queensland,

Australia

Owen Marshall,

University of Tasmania, Australia

*Correspondence:

Anna Hovhanyan

anna.hovhanyan@uni-wuerzburg.de

Thomas Raabe

thomas.raabe@uni-wuerzburg.de

${ }^{\dagger}$ Present address: Eleni Dapergola, Molecular Biosciences, Institute of Biophysical Chemistry, Goethe University Frankfurt, Frankfurt,

Germany

Specialty section:

This article was submitted to

Cell Growth and Division,

a section of the journal

Frontiers in Cell and Developmental

Biology

Received: 17 August 2020

Accepted: 02 February 2021

Published: 05 March 2021

Citation:

Dapergola E, Menegazzi P,

Raabe T and Hovhanyan A (2021)

Light Stimuli and Circadian Clock

Affect Neural Development

in Drosophila melanogaster.

Front. Cell Dev. Biol. 9:595754.

doi: $10.3389 /$ fcell.2021.595754

\section{Light Stimuli and Circadian Clock Affect Neural Development in Drosophila melanogaster}

\author{
Eleni Dapergola ${ }^{1 \dagger}$, Pamela Menegazzi ${ }^{2}$, Thomas Raabe ${ }^{1 *}$ and Anna Hovhanyan ${ }^{1 *}$ \\ ${ }^{1}$ Institute of Medical Radiation and Cell Research, Biozentrum, University of Würzburg, Würzburg, Germany, ${ }^{2}$ Neurobiology \\ and Genetics, Theodor-Boveri Institute, Biozentrum, University of Würzburg, Würzburg, Germany
}

Endogenous clocks enable organisms to adapt cellular processes, physiology, and behavior to daily variation in environmental conditions. Metabolic processes in cyanobacteria to humans are under the influence of the circadian clock, and dysregulation of the circadian clock causes metabolic disorders. In mouse and Drosophila, the circadian clock influences translation of factors involved in ribosome biogenesis and synchronizes protein synthesis. Notably, nutrition signals are mediated by the insulin receptor/target of rapamycin (InR/TOR) pathways to regulate cellular metabolism and growth. However, the role of the circadian clock in Drosophila brain development and the potential impact of clock impairment on neural circuit formation and function is less understood. Here we demonstrate that changes in light stimuli or disruption of the molecular circadian clock cause a defect in neural stem cell growth and proliferation. Moreover, we show that disturbed cell growth and proliferation are accompanied by reduced nucleolar size indicative of impaired ribosomal biogenesis. Further, we define that light and clock independently affect the InR/TOR growth regulatory pathway due to the effect on regulators of protein biosynthesis. Altogether, these data suggest that alterations in InR/TOR signaling induced by changes in light conditions or disruption of the molecular clock have an impact on growth and proliferation properties of neural stem cells in the developing Drosophila brain.

Keywords: Drosophila, neuroblast growth, proliferation, circadian clock, light stimuli

\section{INTRODUCTION}

Endogenous circadian clocks are highly conserved and enable organisms to adjust their physiology and behavior to the day/night cycle. All circadian clocks (1) synchronize to the environment through input pathways, (2) rely on molecular oscillators, which generate the rhythm and thereby keep circadian time, and (3) transmit time information to modulate behavior and physiology through output pathways. Processes modulated by the circadian clock include feeding behavior, locomotor activity, body temperature, hormone level, and metabolic activity (reviewed in Green et al., 2008; Allada and Chung, 2010; Dubowy and Sehgal, 2017).

A hierarchical network of clocks located in different tissues controls these rhythmic processes. The master clock is located in the central nervous system (CNS) and synchronizes organ and tissue clocks. The mammalian master clock resides in the suprachiasmatic nuclei of the hypothalamus comprising about 15,000 clock neurons, whereas in Drosophila, the master clock consists of about 150 clock neurons allocated in several clusters in the lateral and dorsal brain (reviewed in Glossop and Hardin, 2002; Hermann-Luibl and Helfrich-Förster, 2015; Patke et al., 2020). The molecular 
clock machinery is largely conserved across different species and consists of transcriptional-translational feedback loops to maintain the rhythmic cycling of gene expression (reviewed in Patke et al., 2020). Briefly, circadian activators trigger transcription of repressor genes, which, upon translation, feedback to suppress their own transcription. In Drosophila, these circadian activators are Clock (Clk) and Cycle (Cyc), which form a heterodimeric protein complex to trigger the transcription of the circadian repressors, period (per) and timeless (tim), as well as many other target genes. A complex interplay of lightdependent degradation, initiated by the blue light photoreceptor Cryptochrome (Cry), and multiple phosphorylation events regulate the accumulation of Per/Tim heterodimers and their timely translocation into the nucleus to inhibit the transcriptional activity of Clk/Cyc (reviewed in Hardin and Panda, 2013; Dubowy and Sehgal, 2017; Patke et al., 2020). Most core clock components are transcriptional regulators, regulating the expression of approximately $10 \%$ of all genes in a circadian manner (reviewed in Wijnen et al., 2006; Doherty and Kay, 2010; Hughes et al., 2012). Therefore, dysregulation of the circadian system contributes to the pathophysiology of many diseases; most prominent of those are psychiatric disorders and metabolic diseases (reviewed in Bellet and Sassone-Corsi, 2010; Zordan and Sandrelli, 2015; Maury, 2019).

The larval brain clock network is much simpler organized with only nine clock neurons in each hemisphere: five lateral neurons (LNs) and two pairs of dorsal neurons (DN1s and DN2s) (Kaneko et al., 1997). Light sensing is achieved by Cry expression in four out of the five LNs and the DN1s as well as from the larval visual system, the Bolwig organ (BO). The 12 photoreceptor cells of the $\mathrm{BO}$ project are divided into two subtypes according to either rhodopsin 6 (Rh6, sensitive to green light) or rhodopsin 5 ( $\mathrm{Rh} 5$, sensitive to blue light) expression (Malpel et al., 2002; Mazzoni et al., 2005). They project to the four $\mathrm{Cry}^{+}$LNs that act as early pacemaker neurons (PNs), which are responsible for synchronization of the larval brain clock to the light-dark (LD) cycle. Rh5, Rh6, and Cry are each sufficient alone to entrain all larval clock neurons (Keene et al., 2011; Klarsfeld et al., 2011). Rhythmic Per expression in these early PNs throughout development indicated a functional clock network before other clock neurons are integrated at late developmental stages (Kaneko et al., 1997; Liu et al., 2015). Cycling of Per in early PNs is maintained under DD conditions with the peak time of Per expression comparable to LD conditions, indicating a free-running period of approximately $24 \mathrm{~h}$ (Kaneko et al., 1997). Despite the early appearance of a functional clock, no clockcontrolled behavior has been characterized in larvae. A welldefined light-dependent larval behavior is negative phototaxis. Although light avoidance is not regulated in a circadian manner, it requires Rh5 expressing photoreceptors, DN2s, and a single LN (Keene et al., 2011). Even though light during development is dispensable for circadian behavior of individual adults, a single light pulse at larval stages is sufficient to synchronize the activity phases of adult flies reared under constant darkness (Sehgal et al., 1992). A similar effect was observed when the light pulse was given during the second half of embryogenesis (Zhao et al., 2019). Thus, clock neurons in larvae fulfill distinct functions.
Whereas the clock network in the Drosophila CNS regulates circadian rhythms such as locomotor activity, sleep, and eclosion, circadian control of metabolism mostly depends on peripheral oscillators (reviewed in Green et al., 2008; Allada and Chung, 2010). One of the major peripheral clocks in mammals is placed in the liver, which regulates among others metabolism by combining environmental and central clock signals (Lamia et al., 2008). However, limited food conditions might set the peripheral clock without any involvement of the master clock (Damiola et al., 2000; Hara et al., 2001). In Drosophila, the fat body takes over the liver function to regulate feeding behavior and nutrient storage (Xu et al., 2008). In response to nutritional cues, the larval fat body generates mitogens to promote cell growth. A prominent example of fat body dependent cellular growth are the neural progenitor cells (neuroblasts, NBs) in the developing CNS (reviewed in Homem and Knoblich, 2012; Hakes and Brand, 2019). Three different types of NBs exist in the CNS, which differ by their division modes: Type 0 , Type I, and Type II (reviewed in Doe, 2017; Hakes and Brand, 2019). Similar to other stem cells, NBs divide asymmetrically to self-renew and to generate a daughter cell. Daughter cells generated by Type 0 NBs directly differentiate into neurons. Division of Type I NBs gives rise to ganglion mother cells (GMCs), which divide once more to generate two neurons. Type II NBs generate intermediate neural progenitors (INPs), which in turn produce GMCs.

Two waves of neurogenesis, embryonic and larval, ensure CNS development and both correlate with changes in neuroblast (NB) size (reviewed in Homem and Knoblich, 2012; Yasugi and Nishimura, 2016). During the embryonic phase, NBs go through a limited number of divisions, diminishing in size with each division until they become quiescent. The four mushroom body (MB) NBs are exceptional because they proliferate throughout development without a quiescence phase (Ito and Hotta, 1992) to generate the neurons of the MBs, important structures for the learning and memory processes (reviewed in Heisenberg, 2003; Cognigni et al., 2018). The second wave of neurogenesis, during larval and pupal stages, starts with the exit of NBs from quiescence in a nutrition-dependent manner (Britton and Edgar, 1998; Chell and Brand, 2010). In contrast to the embryonic NBs, the larval NBs maintain their original size by re-growing after each division. This continues until the end of neurogenesis, where again NBs decrease in size and exit from the cell cycle (Siegrist et al., 2010).

The major growth regulator pathway to trigger exit from NB quiescence and reactivation of proliferation is governed by insulin receptor (InR)/target of rapamycin (TOR) signaling. The pathway is stimulated by insulin-like peptides (ILPs) generated in insulin-producing glial cells, which receive nutritional signals from the fat body (Géminard et al., 2009; Chell and Brand, 2010; Sousa-Nunes et al., 2011). The TOR pathway might be activated independently from the InR pathway via direct cellular nutrient sensing (reviewed in González and Hall, 2017). The interplay between InR and TOR pathways regulates cell growth through a variety of effector proteins at the levels of gene expression, ribosome biogenesis, and protein synthesis (reviewed in Hietakangas and Cohen, 2009; Russell et al., 2011). 
Despite the well-established link between the circadian system and physiological processes, the influence of the circadian clock on neuronal development and therefore its potential impact on neural circuit formation is poorly understood. To investigate such a link, we compared wild-type and clock mutant larvae exposed to different light regimes. First, we showed that disruption of the molecular circadian clock and different light conditions affect NB growth and proliferation. Second, we found a significant reduction of nucleolus size in NBs, indicating that rRNA production and ribosome biogenesis might be disturbed. Based on these findings we analyzed the effect of light and the circadian clock on the InR/TOR growth regulatory pathway. Specifically, gene expression and activity of Akt as a downstream effector kinase of InR signaling, and S6K (RPS6-p70-protein kinase) as a target of TOR and major regulator of nutrient-dependent metabolism and cell growth, were disturbed when the light regime was changed or clock function was abrogated.

\section{MATERIALS AND METHODS}

\section{Fly Stocks and Genetics}

Flies were maintained at $25^{\circ} \mathrm{C}$ on standard cornmeal food in a 12 h light-dark (LD) cycle. Canton Special (CS) was the control and genetic background for $c r y^{01}$ (Dolezelova et al., 2007) and clock mutant flies: per $^{01}$ (Konopka and Benzer, 1971). To mark Type II NBs, the wor-Gal4, ase-Gal80 driver line was used to express UAS-mCD8:GFP (Neumüller et al., 2011). For RNA isolation or protein extraction, after laying eggs, animals were entrained in a $12 \mathrm{~h} \mathrm{LD}$ cycle or shifted into constant darkness until the 3 rd instar larval stage. Relative to Zeitgeber time 0 (ZT0) as the time of lights-on during the LD cycle and circadian time 0 (CT0) as the time corresponding to subjective lights-on during free running in DD, larvae were collected in a ZT0-ZT4-ZT8ZT12-ZT16-ZT20 or CT0-CT4-CT8-CT12-CT16-CT20 schedule and dissected. In the case of per ${ }^{01}$ larvae were always collected at ZT0 and dissected. Isolated larval brains were collected either in TRIzol (for RNA extraction) or in Laemmli buffer (for protein extraction).

\section{Larval Staging}

Immediately after hatching, larvae were collected from apple juice plates at $30 \mathrm{~min}$ intervals and transferred to standard fly food plates with yeast. per $^{01}$ mutants, wild-type larvae under LD and DD light regime, were kept at $25^{\circ} \mathrm{C}$ until they reached the desired age. Before preparation, larval stages were determined by means of spiracle morphology. Preparation of 1st instar larvae (L1) was done $22 \mathrm{~h}$ after larval hatching (ALH), 2nd instar larvae (L2) were $36 \mathrm{~h} \mathrm{ALH}$, and $3 \mathrm{rd}$ instar larvae (L3) were at the wandering stage approximately $120 \mathrm{~h}$ ALH.

\section{Immunohistochemistry}

For immunostainings, larval brains were dissected in PBS (10 mM Na $\mathrm{HPO}_{4}, 2 \mathrm{mM} \mathrm{KH} \mathrm{PO}_{4}, 2.7 \mathrm{mM} \mathrm{KCl}, 137 \mathrm{mM}$ $\mathrm{NaCl}$ ) and fixed on ice for $25 \mathrm{~min}$ in PLP solution (2\% paraformaldehyde, $10 \mathrm{mM} \mathrm{NaIO}$, $75 \mathrm{mM}$ lysine, $30 \mathrm{mM}$ sodium phosphate buffer, $\mathrm{pH}$ 6.8). All washings were done in
PBT (PBS plus 0.3\% Triton X-100). After blocking in PBT containing $3 \%$ normal goat serum for $2 \mathrm{hr}$, brains were incubated overnight with combinations of the following primary antibodies: rabbit anti-protein kinase C (anti-PKC) (1:1000; clone C20, Santa Cruz Biotechnology, Dallas, TX, United States), rabbit anti-phospho-histone H3 (1:2500; Merck Millipore, Burlington, MA, United States), rat anti-Miranda (1:300; clone CD\#57E9BG5AF4, Abcam, Cambridge, United Kingdom), mouse antiMiranda (1:20; F. Matsuzaki, Kobe, Japan), rabbit anti-Nop5 (1:600; G. Vorbrüggen, Göttingen, Germany), chicken anti-GFP (1:1000; Abcam), rabbit anti-Asense (1:400; F. Diaz-Benjumea, Madrid, Spain), guinea pig anti-Deadpan (1:1000; J. Knoblich, Vienna, Austria), mouse anti-Dachshund (1:10; clone mAbdac23, Developmental Studies Hybridoma Bank [DSHB], Iowa City, IA, United States), rabbit anti-Tailless (1:600; J. Reinitz, Chicago, Illinois, United States), guinea pig anti-lamin DmO (1:300; G. Krohne, Würzburg, Germany), mouse anti-Bruchpilot (1:30; clone nc82, DSHB). Alexa Fluor 546-Phalloidin (1:100; Molecular Probes, Thermo Fisher Scientific, Waltham, MA, United States) was used to mark F-actin. Secondary antibodies were conjugated with Alexa Fluor 488 (Molecular Probes), Cy3, Cy5, or DyLyte488 (Dianova, Hamburg, Germany). After extensive washing in PBT, brains were embedded in Vectashield using distance holders to avoid brain deformation. Confocal images were collected with a Leica SPE microscope. Image processing was done with ImageJ 1.46r software (NIH, Bethesda, MA, United States).

Neuroblasts were visualized by aPKC or Miranda, nucleoli by Nop5 antibodies. The freehand selection tool of ImageJ 1.46r software was used for measuring $\mathrm{NB}$ and nucleolar areas. This allowed to include spherical shaped metaphase NB and the more irregular shaped NB at other cell cycle stages in our analysis. Data were blinded and analyzed independently by at least two persons.

To determine the brain volume of 3rd instar larvae $(120 \mathrm{~h}$ $\mathrm{ALH})$, the average brain lobe diameter was calculated from two orthogonal diameters taken across the brain lobe at its largest dimension, and volume (V) was calculated as $V=4 / 3 \pi r^{3}$. Brain volume was the sum of volumes of the two brain lobes. Adult central brain (CB), optic lobe (OL), and whole brain size measurements of the different genotypes were scored blind with the freehand selection tool of ImageJ 1.46r software.

\section{Neuroblast Proliferation}

The anti-Tailless antibody was used to specifically mark MB NB and derived GMCs (Kurusu et al., 2009). Type II NBs and their lineages were marked with GFP by using UAS-mCD8:GFP line under the control of the wor-Gal4, ase-Gal80 driver line (Neumüller et al., 2011). To distinguish different maturation stages of INPs within the Type II NB lineage, anti-Dpn and antiAse antibodies were used (Bowman et al., 2008; Walsh and Doe, 2017). MB NB derived GMCs and mature INPs (mINPs) were counted with the Amira ${ }^{\circledR}$ software using the Landmark selection.

\section{RNA Isolation and RT-qPCR Analysis}

Wild type and per $^{01}$ mutant larvae were collected in PBS and placed on ice. Dissections of larvae were done within $30 \mathrm{~min}$ and brains were collected in TRIzol ${ }^{\circledR}$ reagent for total RNA extraction according to the manufacturer's instruction (Ambion ${ }^{\circledR}$, Thermo 
Fisher Scientific, Waltham, MA, United States). First-strand cDNA was synthesized from $2 \mu \mathrm{g}$ of RNA using High-Capacity cDNA Reverse Transcription Kits (Applied Biosystems, Thermo Fisher Scientific, Waltham, MA, United States). RT-qPCR was done using PowerUp ${ }^{\mathrm{TM}} \mathrm{SYBR}^{\mathrm{TM}}$ Green Master Mix (Applied Biosystems) on a StepOnePlus ${ }^{\mathrm{TM}}$ (Applied Biosystems) realtime thermal cycler. Reaction mixtures contained $300 \mathrm{nM}$ of oligonucleotides. RT-qPCR conditions were $2 \mathrm{~min} 50^{\circ} \mathrm{C}$ and 2 min $95^{\circ} \mathrm{C}$ holding steps, followed by 40 cycles of $15 \mathrm{~s} 95^{\circ} \mathrm{C}$ and $1 \mathrm{~min} 60^{\circ} \mathrm{C}$. Results were expressed as fold change in expression of the treated sample in relation to untreated samples and relative to the reference gene $r p 49$. Mean \pm SEM was calculated at least from triplicate experiments from each of the three independent biological samples per genotype or different light regime.

The following primers were used to amplify the cDNA of target genes:

Akt: forward 5'-ACAGATCTAGTGTTGAAAAAAATATA CCG-3'

reverse $5^{\prime}$-ATGTCTCCTTGGTAGCTGAACTGCG-3', $S 6 k$ : forward 5' $5^{\prime}$ TTCTTAGAGGATACCACATGCTTC-3' reverse $5^{\prime}$-TGGTCAAAATTTCAGGTGCCATGTAC-3', rp49: forward 5'-GCCCAAGATCGTGAAGAAGC-3' reverse 5' -CGACGCACTCTGTTGTCG-3'.

\section{Western Blot}

Lysates from wild-type or per $^{01}$ larval brains were sonicated in $2 \mathrm{x}$ Laemmli, separated by SDS-PAGE and transferred to PVDF membranes (Amersham ${ }^{\mathrm{TM}}$ Hybond $^{\mathrm{TM}} \mathrm{P} 0.45$ PVDF, GE Healthcare Life science, Chicago, IL, United States). Blots were incubated overnight at $4^{\circ} \mathrm{C}$ with the following antibodies: rabbit anti-phospho-Akt (Ser473) (1:1000; clone D9E, Cell Signaling, Danvers, MA, United States), rabbit anti-Akt (1:1000, Cell Signaling), rabbit anti-phospho-Drosophila-p70 S6k (1:1000; Cell Signaling), mouse anti- $\alpha$-Tubulin (1:5000, clone NDM1A, Merck, Darmstadt, Germany). After incubation with HRP-coupled secondary antibodies, signal detection was done with the ECL Plus detection reagents (GE Healthcare Life Science, Buckinghamshire, United Kingdom) and a ChemoCam ECL Imager equipped with a 16 bit camera (Intas, Göttingen, Germany). Exposure times were adjusted to allow for quantification of signal intensities within the dynamic range of the camera system.

\section{Data Analysis}

Nucleolar size, cell size, and number of progenies were analyzed by Statistica $v$. 9. Distributions of variables did not deviate significantly from normality (Kolmogorov-Smirnov test; $P>0.2$ ). A one-way analysis of variance (ANOVA) was performed for statistical analysis. Axis lengths and areas were considered as dependent variables, and the strain (wild type versus mutant or LD light regime versus DD) was considered as an independent variable.

To calculate the significance of gene expression differences between experimental groups, statistical analyses were performed using the Mann-Whitney- $U$-test (Origin Pro9.0.0 b45 software). For multiple testing within one data set, the level of significance was adjusted with the Bonferroni correction factor. Graphs are presented as mean \pm the $\max$ and min size distribution or \pm SEM, asterisks depict the level of statistical significance **** $p \leq 0.00001$ and ${ }^{* * *} p \leq 0.0001,{ }^{* *} p \leq 0.001$, and ${ }^{*} p \leq 0.01$. Graphs were generated in Prism 6.

\section{RESULTS}

\section{Circadian Clock and Light Independently Control Cell Growth in the Larval Brain}

At all developmental stages, animals sense and respond to changes in both external and internal conditions. The combination of this information regulates behavior and metabolism to benefit from available resources and to maintain cellular homeostasis. Metabolism is important for the proper growth and development of an organism (reviewed in Koyama et al., 2020) and many aspects of metabolism and cellular physiology are controlled by endogenous circadian rhythms (reviewed in Green et al., 2008; Bellet and Sassone-Corsi, 2010; Shi and Zheng, 2013). CNS development of Drosophila is a prominent example to investigate mechanisms, how an organism copes with changes in environmental conditions, e.g., the nutritional status. Nutritional signals after larval hatching are important for NB growth and reactivation of proliferation after the quiescence phase, whereas reduction in NB growth and proliferation terminates neurogenesis during early pupal stages (Siegrist et al., 2010; Spéder and Brand, 2014; reviewed in Lanet and Maurange, 2014). To look for a potential impact of the circadian clock on NB growth, we investigated whether disruption of the circadian clock influences the size of central brain (CB) NBs during development. Moreover, we asked whether light, independently from the clock, also affects cell growth. We measured NB sizes in 3rd instar larval brains of wild type and per $^{01}$ mutants (which lack a functional clock) grown under light-dark (LD) condition. NBs were marked with specific markers (aPKC and Miranda, Figure 1A). Wild type flies were also kept in constant darkness (DD, where the molecular clock still maintains a rhythm of approximately $24 \mathrm{~h}$ without being reset by light stimuli) and constant light (LL, where the circadian clock is not functional anymore due to the constant activation of the photopigment Cry). In addition, to control for effects of the light-dark cycles independent of resetting of the molecular oscillator by Cry, we included $c r y^{01}$ mutants in our experiments. We found significantly smaller NBs in larvae lacking a functional clock, namely per ${ }^{01}$ mutants and wild type kept under LL, respectively, DD conditions, as well as in larvae with a functional oscillator but unable to reset it by light transitions $\left(c r y^{01}\right.$ mutants) (Figures 1B,C). If light and clock independently affect cell growth, the combination of clock disruption and absence of light should enhance the observed cell size defect. Therefore, we analyzed $\mathrm{CB}$ NB sizes from per $^{01}$ mutant $3 \mathrm{rd}$ instar larvae kept under DD conditions. Indeed, the average size of NBs was further reduced in comparison to either per $^{01}$ or wild-type larvae kept in DD, although reduction was neither synergistic nor additive (Figure 1D). In combination with the NB size reduction observed in $c r y^{01}$ mutants and animals kept under LL condition, this indicated at least partial independent effects 

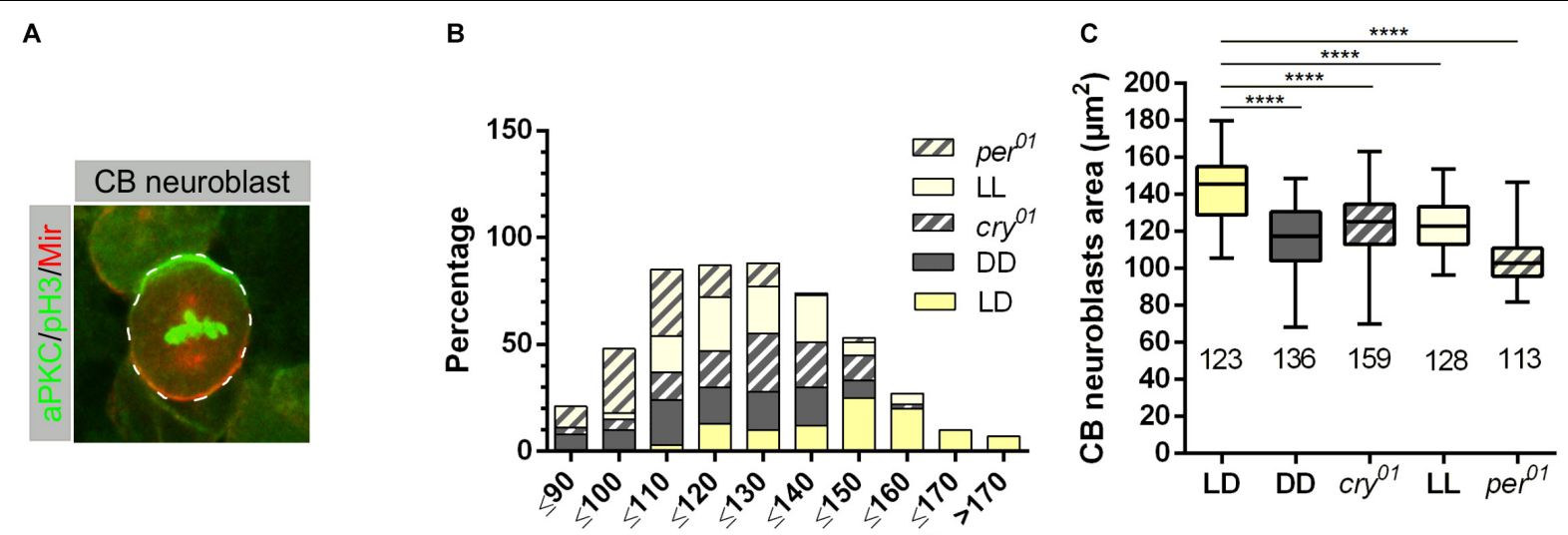

Neuroblast area $\left(\mu \mathrm{m}^{2}\right)$
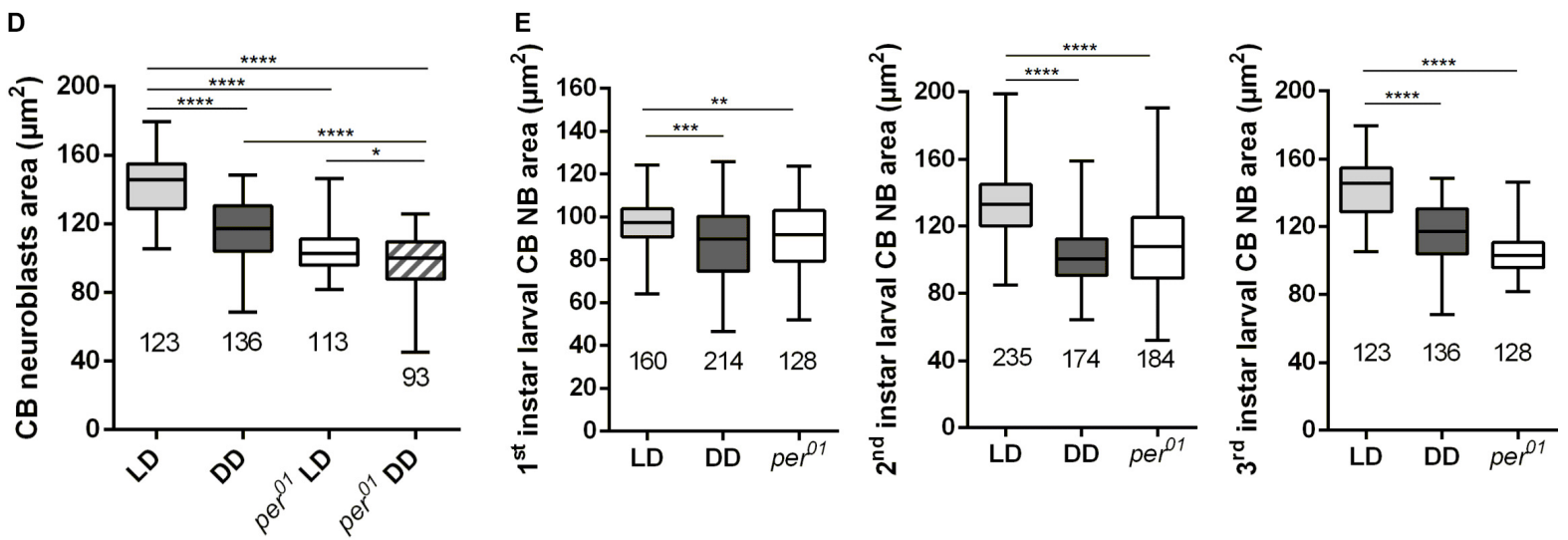

FIGURE 1 | Light and the circadian clock control neuroblast size. (A) Representative example of a central brain (CB) neuroblast (NB) labeled with aPKC (green) and Miranda (red) and phospho-histone $\mathrm{H3}$ (pH3, green) as a mitotic chromatin marker. (B) CB NBs size distribution of wild-type larvae grown under 12:12 h dark/light (LD), constant light (LL), and constant darkness (DD). LD conditions were used for cry ${ }^{01}$ and per ${ }^{01}$ mutant animals. NBs in wild type mainly distribute within 120 to $170 \mu \mathrm{m}^{2}$, NBs in wild-type larvae (DD) and per ${ }^{01}$ mutants range between 90 and $140 \mu \mathrm{m}^{2}$, whereas NB cell size for larvae grown under LL condition and cry ${ }^{01}$ distribute between 110 and $150 \mu \mathrm{m}^{2}$. The number of measured NB for each genotype and light conditions are indicated in (C). (C) Average NBs sizes of all experimental groups shown in (B) $\left(^{* * * *} p<0.00001\right)$. (D) The NB size defect in per ${ }^{01}$ mutant larvae is enhanced under DD conditions $\left({ }^{* * * *} p<0.00001,{ }^{*} p<0.01\right)$. (E) NB size defects are observed throughout larval development for wild type (DD) and per ${ }^{01}\left({ }^{* * *} p<0.00001,{ }^{* * *} p<0.0001,{ }^{* *} p<0.001\right)$. At least 10 brains were analyzed for each genotype and different light condition. The number of measured NBs are indicated below each box plot.

of light and clock on cell growth. As the strongest effects were observed in per ${ }^{11}$ mutants and wild type grown under DD condition, all further experiments were performed using these two experimental groups.

In order to distinguish whether the observed growth defect exists from the beginning of the second wave of neurogenesis starting with larval hatching or it is a later developmental effect, we conducted a staging experiment by measuring the size of $\mathrm{NBs}$ at $22 \mathrm{~h}$ (1st instar), $36 \mathrm{~h}$ (2nd instar), and $120 \mathrm{~h}$ (3rd instar) after larval hatching (ALH). The late 1st instar larval stage was chosen because NBs are sequentially released the quiescence phase starting in early 1st instar larvae (Ito and Hotta, 1992). A significant NB cell size defect was already observed in late 1st instar larvae, which became much more pronounced at later larval stages (Figure 1E).

Since NB reactivation in early 1 st instar larvae is accompanied by cell growth as a prerequisite for proliferation, the observed cell size defect could be a consequence of a delay in reactivation.
Quiescent NBs are arrested either in G0 or in G2 phase of the cell cycle. Within a time window of $48 \mathrm{~h}$ ALH, NBs arrested in the G2 stage reactivate earlier compared to G0 NBs (Otsuki and Brand, 2018). The percentage of metaphase NBs was used as a proxy for proliferation activity of the whole NB population. Whereas the number of metaphase NBs in 1 st (22 h ALH) and 2nd (36 h ALH) instar larvae were comparable between wild type animals (LD and DD conditions) and the per ${ }^{11}$ mutant, we observed a $20-30 \%$ reduction in the number of metaphase NBs in 3rd instar larval brains (120 h ALH) from wild type (DD) and the per ${ }^{01}$ mutant (Figure 2A). This effect was further enhanced when per $^{01}$ animals were kept in DD (Figure 2B). Thus, NB which became reactivated until $36 \mathrm{~h}$ ALH started to proliferate in a wild type manner in both experimental groups.

Given the pronounced NB cell size defect at all larval stages, the question arises whether animals with a disrupted clock or grown under DD condition show a developmental delay at the organismic level. Using the hatching of 1st instar larvae as a 

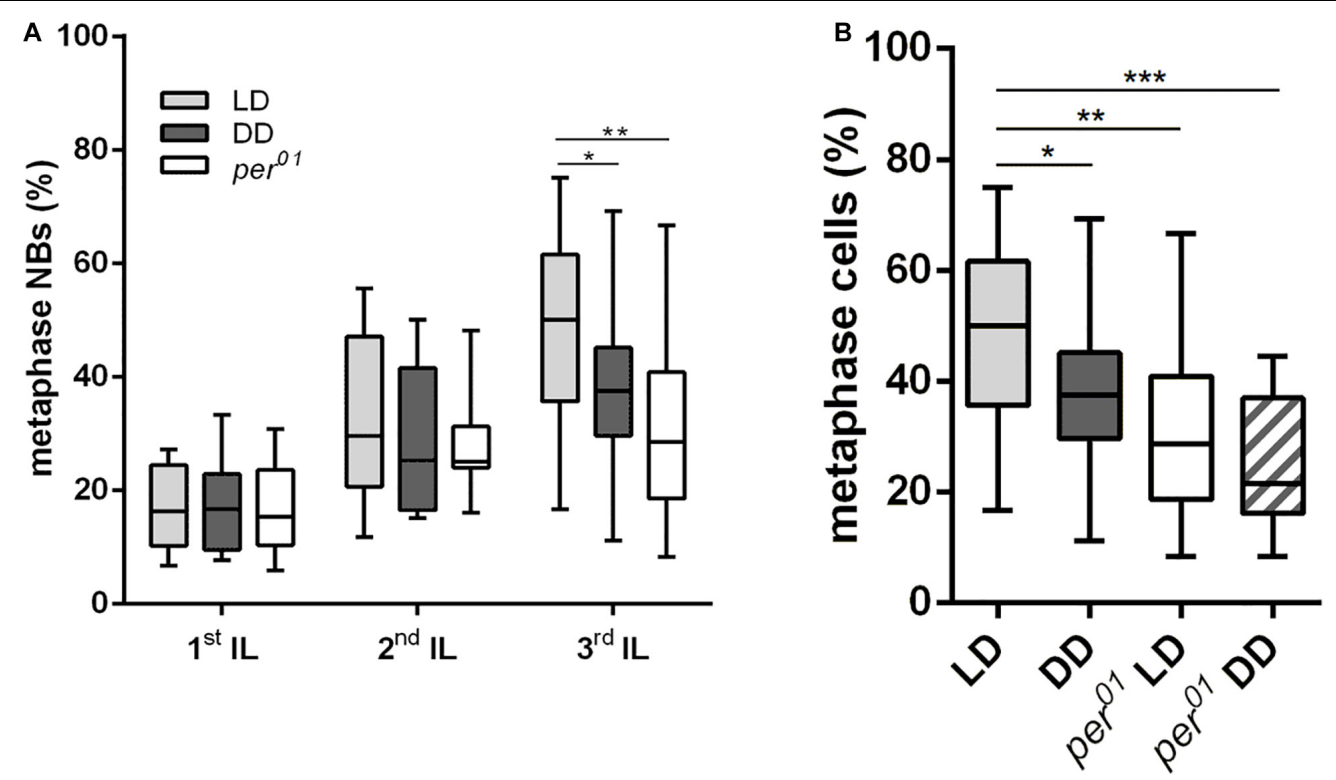

FIGURE 2 | Effects of the circadian clock and light on neuroblast division rates. (A) The number of metaphase NB in relation to the overall NB number was determined in 1st, 2nd, and 3rd instar larval (IL) central brains. Compared to wild type (LD), the DD condition and the per ${ }^{01}$ mutant caused a significant reduction of dividing NBs only in 3rd instar larval brains $\left({ }^{*} p<0.01,{ }^{* *} p<0.001\right)$. (B) The number of metaphase NBs in 3rd instar larval brains was further decreased when per ${ }^{01}$ larvae were kept in DD (*** $p<0.0001)$. At least 16 brains were analyzed for each larval stage and experimental condition.

starting time point, it became evident that pupation time points for all genotypes are largely comparable, with a minor delay of DD kept animals (Supplementary Figure 1). Correspondingly, there was no obvious difference in the appearance of wandering 3 rd instar larvae for wild type and $p^{01}$, but an approximately 1 day delay in the appearance of wild type larvae grown under DD conditions (data not shown).

In summary, our findings provided evidence that not only a functional endogenous clock but also light as an environmental factor are needed to control proper NB size, which correlates with impaired proliferation at the end of larval life.

\section{Circadian Clock and Light Are Required for Cell Proliferation}

The coordination between cell growth and proliferation is important for the proper development of tissues (reviewed in Homem et al., 2015). Since we found evidence that the endogenous clock and light have an impact on the growth of NBs, we assumed that the generation of progeny cells by NBs might be affected by the circadian clock and light. Because this issue is difficult to address for the whole heterogeneous NB population, we determined the number of progeny cells for two well-characterized subtypes of NBs: mushroom body (MB) NBs and Type II NBs. The number of these NBs are only four, respectively, eight in each hemisphere, and specific markers are available to label these NBs and their progeny cells. MB and Type II NBs have different modes of division. MB NBs follow the Type I NB division mode to give rise to a self-renewing $\mathrm{NB}$ and a GMC, which divides one more time to generate two neurons (Figure 3A). Type II NBs divide asymmetrically to generate a self-renewing NB and a transient amplifying cell called immature intermediate neural progenitor (iINP), which by transcriptional changes becomes a mature INP (mINP). Each mINP divides asymmetrically three to five times to form another mINP and a GMC, giving rise to two neurons (Figure 3B). To identify $\mathrm{MB}$ NBs and their progenies, in addition to Miranda (Mir) as a general NB marker, an anti-Tailless (Tll) antibody was used to label MB NBs and derived GMCs, whereas Dachshund (Dac) is expressed in $\mathrm{MB}$ neurons (Figure 3A; Kurusu et al., 2000, 2009; Kraft et al., 2016). To label the Type II NB lineage, UAS-mCD8:GFP was expressed under the control of wor-Gal4, ase-Gal80 (Neumüller et al., 2011). Additionally, anti-Deadpan (Dpn) and anti-Asense (Ase) antibodies were used to distinguish between progenies of Type II NBs, as Dpn is expressed only in Type II NBs and mINPs, whereas Ase labels iINPs, GMCs and is also co-expressed with Dpn in mINPs (Figure 3B; Bowman et al., 2008; Walsh and Doe, 2017). First, we verified whether the observed general NB growth defect is also evident for $\mathrm{MB}$ and Type II NBs. Cell size measurements revealed that both types of NBs in 3rd instar larval brains were significantly reduced in size under DD conditions and in per ${ }^{01}$ animals when compared to the wild type LD control (Figures 3C,E). This finding supports our conclusion that light input and a functional clock are required for all NBs to maintain proper cell size. To determine whether the observed reduced cell size is restricted to the NB population, we also examined the cell sizes of MB NB derived GMCs and Type II NB derived mINPs. The size of the analyzed progenies was comparable among the different experimental groups (Supplementary Figure 2).

For analysis of the proliferation capacity of $\mathrm{MB}$ or Type II NBs, we counted $\mathrm{Tll}^{+}$GMCs and $\mathrm{Dpn}^{+} / \mathrm{Ase}^{+}$mINPs generated 


\section{A}

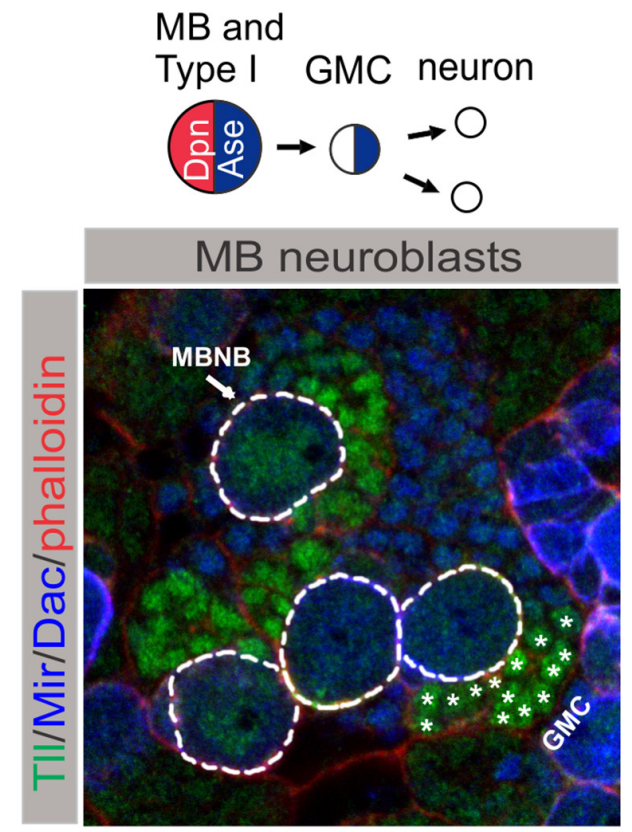

B

Type II iINP iINP mINPGMC neuron

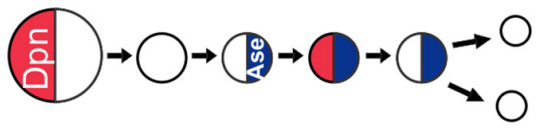

Type II neuroblasts

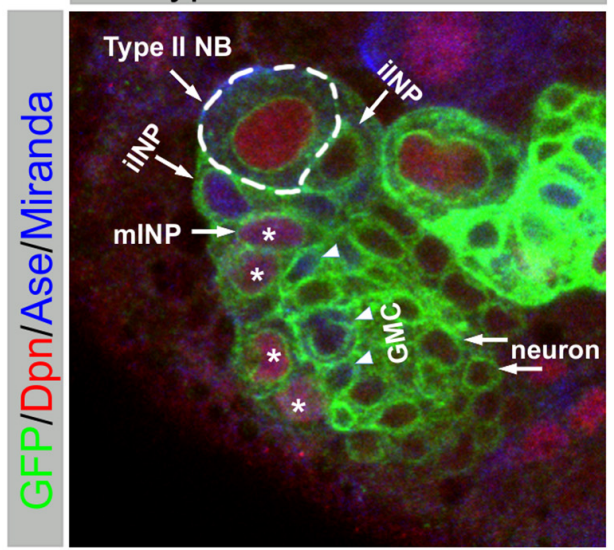

C

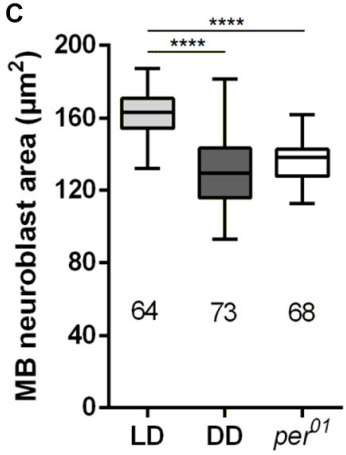

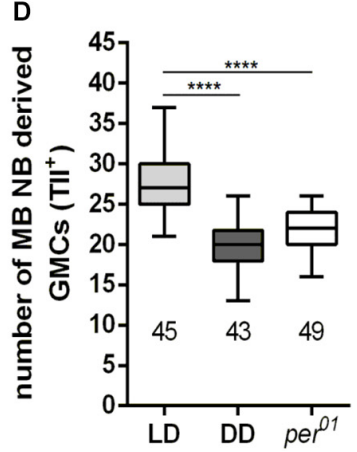
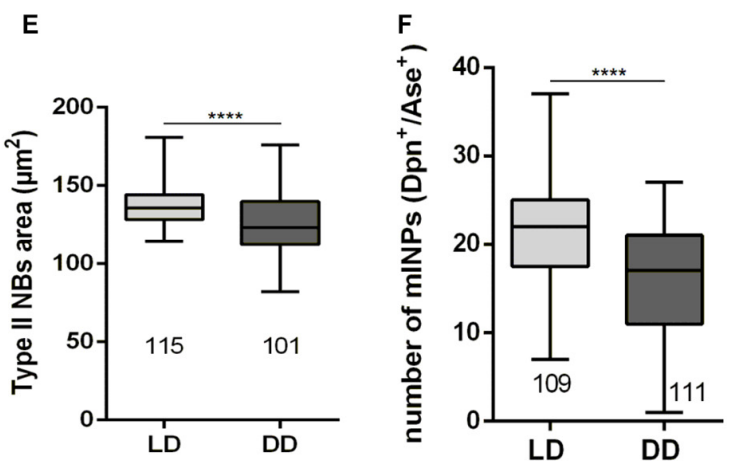

FIGURE 3 | Effect of light and the molecular clock on mushroom body and Type II neuroblast sizes and proliferation. (A) Miranda (Mir blue) was used as a NB marker, Tailless (TII green) marks mushroom body (MB) NBs and derived GMCs (*), Dachshund (Dac blue) labels MB neurons in wandering 3rd instar larval brains. Phalloidin (red) was used as a marker for cortical actin. (C) The decreased MB NB size under DD conditions and in per ${ }^{11}$ mutant larvae was accompanied by a reduced number of GMCs (D). (B) Type II NB lineages were marked with GFP (green) expressed under wor-Gal4, ase-Gal80 control. In addition to the cortical NB marker Miranda (blue), nuclear Dpn (red) and Ase (blue) were used to distinguish immature INPs (ilNP) and mature INPs (mINP*). (E) Type II NB size as well as the number of mINPs $\left(\mathrm{Dpn}^{+} / \mathrm{Ase}^{+}\right) \mathbf{( F )}$ were significantly reduced in larvae under DD conditions. At least 10 brains were analyzed for each genotype or light condition. Data represent the mean obtained from the number of measured NBs or counted progenies \pm the max and min size distribution. The number of measured NBs or progeny cells are indicated below of each box plot $(* * * * 0<0.00001)$.

from single NBs, respectively. In both cases, we observed a pronounced reduction in the number of GMCs and mINPs under DD conditions and in per $^{01}$ animals (Figures 3D,F).

Since the GMCs and INPs are the precursors for neurons, one consequence could be a reduced brain size. To address this question, 3rd instar larval brain volumes were determined from stainings with antibodies against the synaptic protein Bruchpilot (Brp) to label neuropile structures and against the nuclear membrane protein Lamin to outline the cellular cortex. We observed a significant reduction in brain size for wild type larvae grown under DD conditions, but not for the per ${ }^{01}$ mutant, when compared to brains from wild type (LD) (Supplementary Figure $3 \mathrm{~A}$ ). For the DD condition, this corresponded to the reduced number of progeny cells derived from MB and Type II NBs and also indicated a more general effect on proliferation of NBs. To determine whether DD conditions also have an impact on final brain size in the adult, we measured the area of the $\mathrm{CB}$, the optic lobes (OL), and the complete brain. Brains were stained for Brp and Lamin and reconstructed from optical sections before outlining the borders. In contrast to the analysis of larval brains, DD conditions had no negative influence on adult brain size, which was comparable to wild type (LD) and $p e r^{01}$ mutant brains 
(Supplementary Figure 3B). Thus, animals can cope at least to a large degree with changes of light conditions or a nonfunctional clock to reach normal adult brain size despite a defect at the level of NBs.

\section{Effect of Endogenous Clock and Light on Nucleolar Size and Transcriptional Activity}

A possible explanation for the compromised proliferation observed for $\mathrm{MB}$ and Type II NBs (Figures 3D,F) could be the inability of NBs to produce enough proteins to fulfill the requirements of the cell to gain appropriate mass and size before division. The efficiency of protein biosynthesis depends on proper nucleolar function as a site for rRNA transcription/processing and assembly of ribosomes. Thus, the nucleolus is a critical player to maintain cell homeostasis and directly affects cell growth and proliferation. Nucleolar size positively correlates with the amount of rRNA biosynthesis (reviewed in Stępiński, 2018). Specifically, it is directly related to RNA polymerase I transcriptional activity and nucleolar size is highest at the end of the G2 phase, before cell division takes place (Maszewski and Kwiatkowska, 1984, reviewed in HernandezVerdun, 2011). To verify, whether light or a disturbed circadian clock influence RNA synthesis, we compared the size of NBs nucleoli in per ${ }^{01}$ mutant and wild type grown under DD and LD conditions. Interphase NBs were marked using aPKC and Mir, Lamin outlines the nuclear membrane, and Nop5 was used as a nucleolar marker (Figure 4A).

Nucleolar sizes of NBs in per $^{01}$ mutant and wild-type larvae grown under DD conditions were significantly smaller compared to wild type (Figure 4B). Moreover, classification of nucleolar size as large $\left(\geq 9 \mu \mathrm{m}^{2}\right)$, intermediate $\left(\geq 6 \mu \mathrm{m}^{2}\right)$, or small $\left(<6 \mu \mathrm{m}^{2}\right)$ revealed the strongest effect in wild-type larvae grown under DD conditions, where $72 \%$ of the nucleoli were small and only $27 \%$ of them reached intermediate size. In per $^{01}$ mutants, NB nucleolar sizes were equally (46\%) distributed between intermediate and small sizes, whereas the nucleolar size of wild type in LD conditions was mostly distributed between large (42\%) and intermediate (48\%), with only $10 \%$ nucleoli being small (Figure 4C).

Taken together, these results provided evidence that the capability of NBs of per ${ }^{01}$ mutants and wild-type animals grown under DD to synthesize proteins might be impaired, which finally could result in the observed cell growth defects.

\section{InR and TOR Signaling Are Controlled by the Light Regime and the Circadian Clock}

Reactivation of NBs after quiescence and their regrowth after each division during larval development requires the InR/TOR signaling pathways (Chell and Brand, 2010; Sousa-Nunes et al., 2011). In general, impaired InR/TOR signaling results in reduced cell size and proliferation (Zhang et al., 2000, reviewed in Russell et al., 2011). Regulation of protein kinase p70 S6K (S6K) catalytic activity, a downstream target of the TOR pathway, is a major mechanism to control metabolism and cell growth due to its role in ribosome biogenesis and protein translation. Protein kinase Akt is an important signaling molecule in the InR pathway and one upstream regulator of TOR (reviewed in Hietakangas and Cohen, 2009; Russell et al., 2011).

Our findings with regard to NB and nucleolar size reduction and the role of nucleolus in the maintenance of cell homeostasis led to the assumption that light might have a regulatory effect on InR/TOR signaling. Therefore, we tested the impact of light on transcriptional and post-transcriptional regulation of Akt and S6K in 3rd instar larval brains. Quantitative reverse transcription (RT)-PCR analyses showed that $A k t$ and $S 6 k$ mRNA transcriptional levels were reduced at most time points in
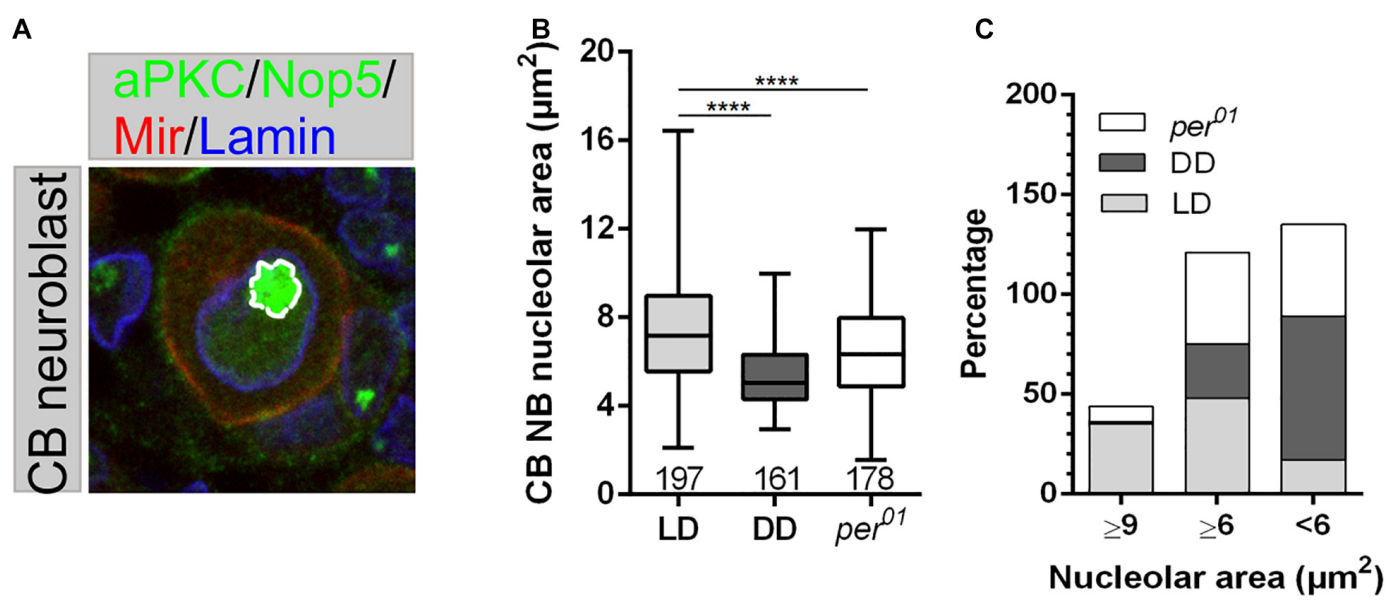

FIGURE 4 | Nucleolar size is affected by light and the circadian clock. (A) Neuroblasts in 3rd instar larval brains were marked for cortical aPKC (green) and Mir (red), the nuclear membrane protein Lamin (blue), and Nop5 (green) as a nucleolar marker. (B) Compared to the control (wild type, LD), central brain NB nucleolar sizes are significantly reduced under DD conditions and in $p e r^{01}$ animals $\left(^{* * *} p<0.00001\right)$. At least 10 brains were analyzed for each genotype and different light condition. Data represent the mean obtained from the number of measured nucleoli \pm the max and min size distribution. The number of analyzed NB are indicated below of each box plot; (C) Nucleolar size distribution of the experimental groups shown in (B). 
DD compared to LD conditions, but differences were below significant levels (Figures 5A,C). Correspondingly, we did not observe any differences for Akt protein total expression level during a period of $24 \mathrm{~h}$ between the two experimental groups (Supplementary Figure 4). For S6K, no antibody was available to test for total protein expression in Drosophila. Although there was no change at transcriptional level for both kinases and total protein expression level at least for Akt, significant differences were evident in phosphorylation of Akt and S6K at most time points under DD conditions. Both kinases showed a significant reduction in phosphorylation of residues as a readout of kinase activity (Figures 5B,D).

We also analyzed the regulatory effect of the circadian clock on gene and protein expression levels of Akt and S6K. Similar to the DD condition, we did not observe differences in the Akt protein level between wild type and per $^{01}$ (Supplementary Figure 5). In contrast to the DD conditions, the RT-qPCR analysis showed that disruption of the circadian clock in per $^{01}$ resulted in reduced expression of $A k t$ and $S 6 k$ mRNAs (Figures 6A,C). In addition, phosphorylation of Akt and S6K were more severely reduced in per $^{01}$ mutant flies in comparison to the DD condition (Figures 6B,D).

These results indicated that activation of two important components of the InR/TOR signaling pathways is under influence of light and the circadian clock. The impairment of signaling by the reduction of phosphorylation of Akt and S6K might lead to cell growth and proliferation suppression.

\section{DISCUSSION}

\section{Regulation of Neuroblast Size and Proliferation by Light and the Circadian Clock}

Metabolic homeostasis relies on accurate circadian timing (reviewed in Green et al., 2008; Eckel-Mahan and Sassone-Corsi, 2013; Maury, 2019). A disrupted circadian clock causes severe disturbances in rhythmic gene expression (reviewed in Bellet and Sassone-Corsi, 2010; Hughes et al., 2012), many of them being involved in metabolism (reviewed in Panda et al., 2002).
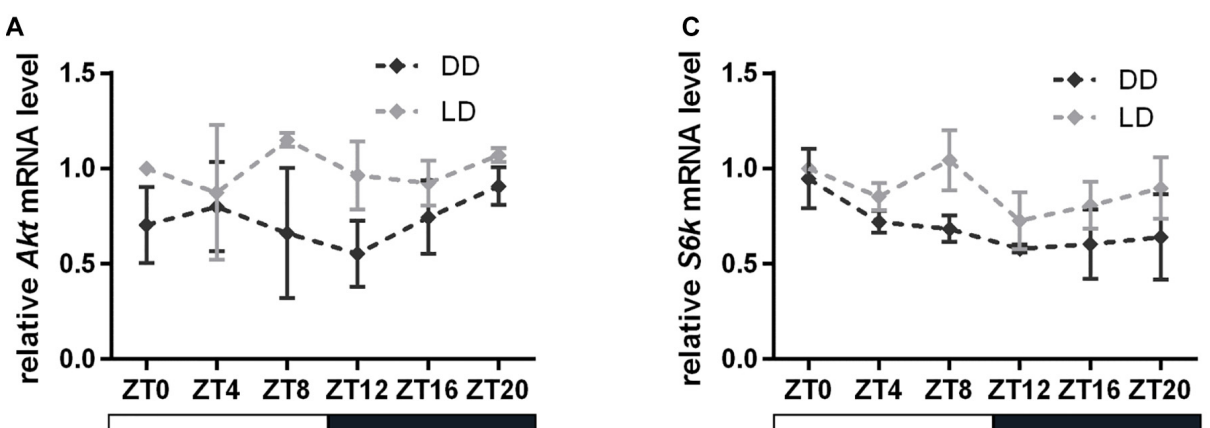

B

ZT 0 ZT 4 ZT 8 ZT 12 ZT 16 ZT 20 LD DD LD DD LD DD LD DD LD DD LD DD

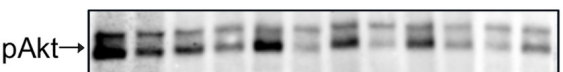

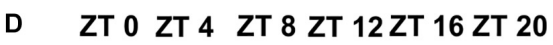
LD DD LD DD LD DD LD DD LD DD LD DD

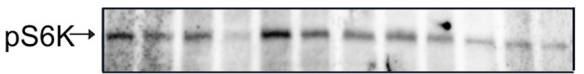

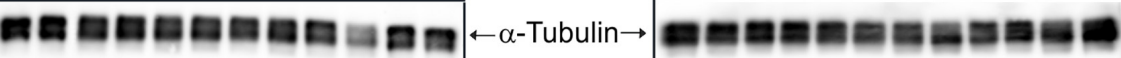
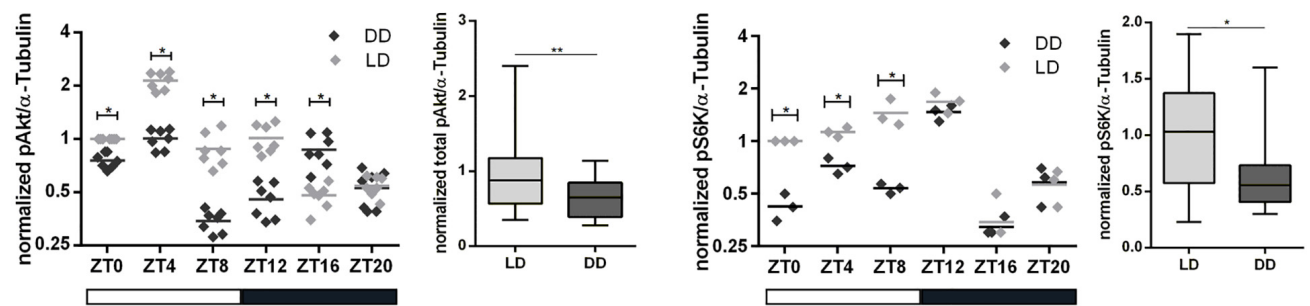

FIGURE 5 | Influence of light on transcription and activation of Akt and S6K. Total RNA and proteins were isolated from wild type 3rd instar larval brains at the indicated Zeitgeber time (ZT). Larvae were grown under LD or DD conditions. (A,C) Gene expression of Akt (A) and S6k (C) at the indicated ZT and light condition. Each time point represents the mean \pm standard error of the mean (SEM) obtained from three biological replicates, each repeated in triplicates. As an internal control, rp49 was used. (B,D) Representative Western blots probed for pAkt (B) and pS6K (D) at the indicated ZT and light condition. $\alpha$-Tubulin was used as a loading control. Dot plots show measurements of Akt and S6K phosphorylation level out of seven and three biological replicates, respectively, normalized to $\alpha$-Tubulin. Box plots display the sum of all time points measurements of Akt and S6K phosphorylation for LD and DD conditions $\left({ }^{* *} p<0.001\right.$ and $\left.* p<0.01\right)$. 

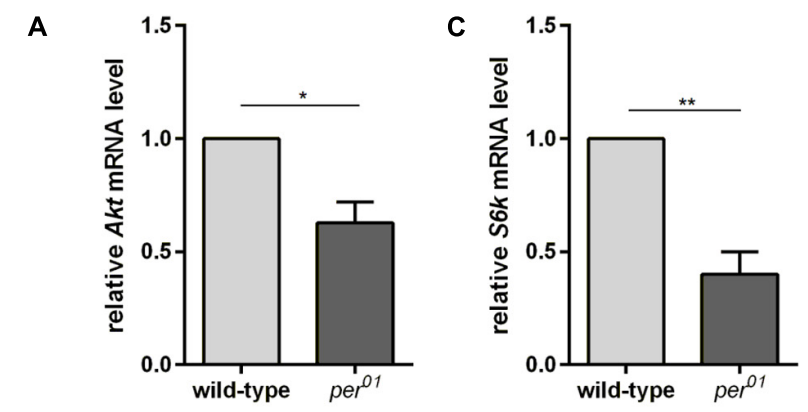

B

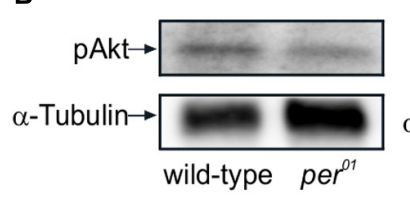

D
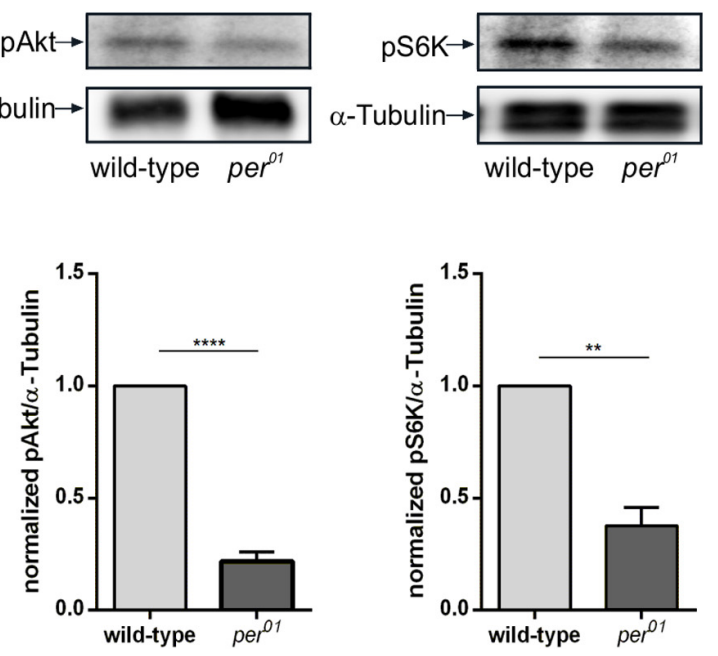

FIGURE 6 | Clock dependent transcription and phosphorylation of Akt and S6K. (A,C) RT-qPCR analysis of Akt (A) and S6k (C) transcription in 3rd instar larval brains from wild type and $p e r^{01}$. Each bar represents the mean \pm standard error of the mean (SEM) obtained from three biological replicates, each repeated in triplicates. As an internal control, rp49 was used $\left({ }^{*} p<0.01\right.$ and ${ }^{* *} p<0.001$, respectively). (B,D) Representative Western blots from 3rd instar larval brain lysates probed for pAKt (B) and pS6K (D). $\alpha$-Tubulin was used as a loading control. Graphs represent measurements of pAkt and pS6K phosphorylation level out of three biological replicates normalized to $\alpha$-Tubulin (mean \pm SEM) $\left({ }^{* * *} p<0.00001\right.$ and $\left.{ }^{* *} p<0.001\right)$.

Furthermore, environmental factors such as light, temperature, or nutrition modulate circadian rhythmicity and synchronize the endogenous clock with the environment. Animals with a disrupted circadian system, caused by exposure to shifted light/dark cycles, show various pathological symptoms including metabolic deficits (Marcheva et al., 2010; Barclay et al., 2012; Mota et al., 2017; Maury, 2019).

The embryonic and postembryonic waves of neurogenesis in Drosophila are separated by a quiescence phase. Release from quiescence requires enlargement of NBs in a nutrition dependent manner before they initiate proliferation. Postembryonic NBs grow and regain their size between each round of division until they shrink again and exit cell cycle at the late larval and pupal stages (Ito and Hotta, 1992; Britton and Edgar, 1998; Truman and Bate, 1988; Maurange et al., 2008; Chell and Brand, 2010; Siegrist et al., 2010). In this study we showed that NB size and proliferation require both clock function and light information. Under DD conditions or absence of a functional clock ( per $^{01}$ ), a NB size defect was observed throughout larval development with stronger defects at later larval stages (Figure 1E). Larvae with a disrupted clock grown under DD condition showed an even more pronounced effect on NB size, which argues for an at least partially independent influence of the circadian system and light on NB growth (Figures 1D, 2B).

Given the correlation between increase of NB size and proliferation at the beginning and decrease at the end of the larval phase of neurogenesis in wild type animals (reviewed in Homem et al., 2015), we also expected to see a proliferation defect in the per $^{01}$ mutant and animals grown under DD condition. Although both experimental groups showed a significant reduction in NB size already in 1st instar larval brains, the percentage of metaphase NBs remained at wild type levels and dropped not before 3rd larval instar (Figure 2A). One explanation is that all NBs enter the cell cycle after release from quiescence as in wild type, but the progressive reduction in NB size limits NB proliferation when they fall below a critical threshold size. An alternative explanation considers that reactivation/growth of G0 arrested NBs takes places until $48 \mathrm{~h} \mathrm{ALH} \mathrm{(Otsuki} \mathrm{and}$ Brand, 2018), whereas our analysis of 2 nd instar larvae was performed at $36 \mathrm{~h} \mathrm{ALH}$. At this time point, proliferation was apparently normal, but we cannot exclude the possibility that reactivation of remaining G0 arrested NBs between 36 and $48 \mathrm{~h}$ ALH was disturbed, causing the observed decrease of metaphase NBs in 3rd instar larval brains from per $^{01}$ and DD kept animals.

A direct effect of light and the endogenous clock on the number of progeny cells generated from a single NB was demonstrated for mushroom body NBs and Type II NBs (Figures 3D,F). Interestingly, neither mushroom body $\mathrm{NB}$ derived GMCs nor Type II NB derived mINPs showed cell size defects (Supplementary Figure 2) arguing for a rather cell-type specific effect of light and the circadian clock on cell size control. Despite the reduced number of progeny cells generated from both types of NBs, an influence on final adult brain size was not evident (Supplementary Figure 3B). However, 3rd instar larval brain size of animals grown under DD condition was significantly reduced in comparison to LD conditions and the per $^{01}$ mutant (Supplementary Figure A). Because 3rd instar larvae were chosen at the same developmental stage just before pupation, brain development under DD conditions is apparently delayed at this stage, and this phenotype becomes at least largely rescued during pupal stages. However, as one caveat, small changes in neuron numbers might not lead to measurable adult brain size differences in our way of analysis. Therefore, it will be necessary to perform labeling and cell counting of neurons derived from individual NB to clarify whether any structure of the adult central brain is affected under DD conditions or in the per $^{01}$ mutant.

Neuroblast reactivation requires nutritional input sensed by the fat body, which in turn activates NB ensheathing glial cells by fat body derived signals (FDS) to produce Insulin-like peptides (ILP). Reactivation of NBs requires ILP signals and amino acid uptake, which stimulate the InR/TOR signaling pathways to 
trigger the first growth of the neuroblast before they resume proliferation (Chell and Brand, 2010; Sousa-Nunes et al., 2011). Additionally, glial cells also express Activin-like peptides (ALPs), which have a mitogenic effect on NBs and are important for stimulating NB division (Zhu et al., 2008). It will be of interest to find out whether expression of ILPs and ALPs are affected under DD condition and in clock mutants.

On the other hand, the peripheral clock in the fat body plays an essential role in feeding rhythmicity and energy storage, thus being responsible for the homeostatic control of the organism. Additionally, light also drives feeding rhythms independent of clock function (Xu et al., 2008). Thus, a major interest would be to elucidate the requirement of the peripheral fat body clock in the regulation of NB growth and proliferation.

\section{Coordination of Cellular Growth Regulatory Pathways by Light and the Circadian Clock}

Neuroblast must gain appropriate mass and size before division, which directly correlates with a high demand for proteins. Major steps for protein biosynthesis are ribosomal RNA synthesis via rDNA transcription, ribosome biogenesis, translation, and posttranslational modification. In consideration of the high metabolic costs of RNA synthesis and ribosome biogenesis, protein biosynthesis represents one of the main metabolic activities in growing and dividing cells. In mammals, the circadian clock coordinates ribosome biogenesis on the transcriptional and translational levels (Jouffe et al., 2013). The signaling pathways regulating these processes are rhythmically activated in a clock-dependent manner. Interestingly, translated mRNAs are mostly involved in ribosome biogenesis (Jouffe et al., 2013). The nucleolus is the site where RNA synthesis and ribosome biogenesis take place, thus the size of the nucleolus correlates with cell growth ability. Our observations that flies under DD condition and flies with depleted clock function have reduced nucleoli (Figures 4B,C) indicated that the observed growth and proliferation defect could be due to a failure of sufficient protein biosynthesis.

The major growth regulatory pathway, InR/TOR signaling, is highly conserved between different organisms (reviewed in Hietakangas and Cohen, 2009). Most of the genes regulated by the TOR signaling pathway are involved in rDNA transcription, ribosome biogenesis, and translation initiation (Guertin et al., 2006; Grewal et al., 2007). Furthermore, Drosophila larvae deficient for TOR show a reduced nucleolar size and a developmental arrest (Zhang et al., 2000). The observed cell size phenotype raised the question, whether light and the circadian clock regulate cell growth through modulation of InR/TOR signaling.

Target of rapamycin signaling is promoted by activated Akt kinase as a downstream target of InR (Gao and Pan, 2001; Inoki et al., 2002; Potter et al., 2002). Independent from InR, the TOR pathway becomes activated through nutritional or amino acid sensing mechanisms (Colombani et al., 2003; Kim et al., 2008; Shim et al., 2013). In both cases, cellular growth is stimulated by phosphorylation of TOR downstream effector proteins. We focused on protein kinase Akt as an upstream regulator of TOR and on $\mathrm{S} 6 \mathrm{~K}$ as a major downstream effector protein kinase. S6K phosphorylates and thereby regulates several proteins essential for $\mathrm{mRNA}$ translation initiation and translation efficiency. Additionally, activated $\mathrm{S} 6 \mathrm{~K}$ plays a role in small ribosome biogenesis by phosphorylation of ribosomal protein S6 (reviewed in Hietakangas and Cohen, 2009; Saxton and Sabatini, 2017).

Here we showed that $A k t$ and $S 6 k$ gene expression in $3 \mathrm{rd}$ instar larval brains were not rhythmic and total Akt and S6K protein levels did not differ significantly between wild type grown under LD and DD within a $24 \mathrm{~h}$ period (Figures 5A,C). However, the kinase activities of Akt and S6K in brains were significantly reduced at several time points when animals were kept in DD (Figures 5B,D). Disruption of the circadian clock in per $^{01}$ animals had an even stronger negative effect on Akt and S6K activity (Figures 6B,D), but also on their gene expression levels (Figures 6A,C). These observations indicate that the decreased NB size might be caused by disturbed Akt activation of TOR resulting in impaired S6K signaling and decreased translation initiation and/or small ribosome subunit biogenesis.

Studies in mammals showed that TOR signaling and the light entrainable circadian clock reciprocal regulate each other, and also an independent food anticipatory clock has an impact on TOR signaling (Cao et al., 2011, 2013; Khapre et al., 2014). Similarly, the peripheral clock in the fat body of flies can be synchronized independently of the central clock by light and via time-restricted feeding (Xu et al., 2011). On the other hand, increasing Akt or TOR activity levels lengthens the circadian period (Zheng and Sehgal, 2010). These examples illustrate the complex interactions between central and peripheral clocks, their mutual relationship with TOR signaling, the impact of environmental factors such as periodic food availability on behavior and physiology, and vice versa the influence of metabolic dysregulation on rhythmic behavior.

The survival rate and fitness of an organism are regulated by adjusting developmental growth, metabolism, and behavior to environmental changes. Our current findings provided evidence that TOR signaling is influenced by clock dependent mechanisms and light. Natural light is important for a living organism to generate physiological and behavioral traits synchronized not only to light-dark daily rhythm but also to seasonal changes. Hence, light intensities as well as photoperiodicity might have an effect on neural circuit formation by affecting neural stem cell growth and proliferation.

\section{DATA AVAILABILITY STATEMENT}

All datasets presented in this study are included in the article/Supplementary Material.

\section{AUTHOR CONTRIBUTIONS}

$\mathrm{AH}$ designed the research. ED and $\mathrm{AH}$ performed the research and prepared the figures. ED, $\mathrm{AH}$, and $\mathrm{PM}$ analyzed the data. 
$\mathrm{AH}$ and TR wrote the manuscript with support of all other authors. All authors contributed to the article and approved the submitted version.

\section{FUNDING}

This work was initially funded by the German Research Foundation (DFG, SFB 1047 "Insect Timing," Project A6 TR), German Excellence Initiative to the Graduate School of Life Sciences, University of Würzburg (GSC106 - AH), and Open Access Publication Fund of the University of Würzburg.

\section{REFERENCES}

Allada, R., and Chung, B. Y. (2010). Circadian organization of behavior and physiology in Drosophila. Annu. Rev. Physiol. 72, 605-624. doi: 10.1146/ annurev-physiol-021909-135815

Barclay, J. L., Husse, J., Bode, B., Naujokat, N., Meyer-Kovac, J., Schmid, S. M. et al. (2012). Circadian desynchrony promotes metabolic disruption in a mouse model of shiftwork. PLoS One 7:e37150. doi: 10.1371/journal.pone.0037150

Bellet, M. M., and Sassone-Corsi, P. (2010). Mammalian circadian clock and metabolism - the epigenetic link. J. Cell Sci. 123(Pt. 22), 3837-3848. doi: 10. 1242/jcs.051649

Bowman, S. K., Rolland, V., Betschinger, J., Kinsey, K. A., Emery, G., and Knoblich, J. A. (2008). The tumor suppressors brat and numb regulate transit-amplifying neuroblast lineages in Drosophila. Dev. Cell 14, 535-546. doi: 10.1016/j.devcel. 2008.03.004

Britton, J. S., and Edgar, B. A. (1998). Environmental control of the cell cycle in Drosophila: nutrition activates mitotic and endoreplicative cells by distinct mechanisms. Development 125, 2149-2158.

Cao, R., Anderson, F. E., Jung, Y. J., Dziema, H., and Obrietan, K. (2011). Circadian regulation of mammalian target of rapamycin signaling in the mouse suprachiasmatic nucleus. Neuroscience 181, 79-88. doi: 10.1016/j.neuroscience. 2011.03.005

Cao, R., Robinson, B., Xu, H., Gkogkas, C., Khoutorsky, A., Alain, T., et al. (2013). Translational control of entrainment and synchrony of the suprachiasmatic circadian clock by mTOR/4E-BP1 signaling. Neuron 79, 712-724. doi: 10.1016/ j.neuron.2013.06.026

Chell, J. M., and Brand, A. H. (2010). Nutrition-responsive glia control exit of neural stem cells from quiescence. Cell 143, 1161-1173. doi: 10.1016/j.cell.2010. 12.007

Cognigni, P., Felsenberg, J., and Waddell, S. (2018). Do the right thing: neural network mechanisms of memory formation, expression and update in Drosophila. Curr. Opin. Neurobiol. 49, 51-58. doi: 10.1016/j.conb.2017. 12.002

Colombani, J., Raisin, S., Pantalacci, S., Radimerski, T., Montagne, J., and Léopold, P. (2003). A nutrient sensor mechanism controls Drosophila growth. Cell 114, 739-749. doi: 10.1016/s0092-8674(03)00713-x

Damiola, F., Le Minh, N., Preitner, N., Kornmann, B., Fleury-Olela, F., and Schibler, U. (2000). Restricted feeding uncouples circadian oscillators in peripheral tissues from the central pacemaker in the suprachiasmatic nucleus. Genes Dev. 14, 2950-2961. doi: 10.1101/gad.183500

Doe, C. Q. (2017). Temporal patterning in the Drosophila CNS. Annu. Rev. Cell Dev. Biol. 33, 219-240. doi: 10.1146/annurev-cellbio-111315-125210

Doherty, C. J., and Kay, S. A. (2010). Circadian control of global gene expression patterns. Annu. Rev. Genet. 44, 419-444. doi: 10.1146/annurev-genet-102209163432

Dolezelova, E., Dolezel, D., and Hall, J. C. (2007). Rhythm defects caused by newly engineered null mutations in Drosophila's cryptochrome gene. Genetics 177, 329-345. doi: 10.1534/genetics.107.076513

\section{ACKNOWLEDGMENTS}

We would like to thank Vahan Serobyan for discussion and comments on the manuscript. We would also like to thank Kjara Sophia Pilch for assistance in RT-qPCR experiments. The preprint of the current manuscript is posted on bioRxiv: https:// biorxiv.org/cgi/content/short/2020.08.07.241208v1.

\section{SUPPLEMENTARY MATERIAL}

The Supplementary Material for this article can be found online at: https://www.frontiersin.org/articles/10.3389/fcell.2021. 595754/full\#supplementary-material

Dubowy, C., and Sehgal, A. (2017). Circadian rhythms and sleep in Drosophila melanogaster. Genetics 205, 1373-1397. doi: 10.1534/genetics.115.185157

Eckel-Mahan, K., and Sassone-Corsi, P. (2013). Metabolism and the circadian clock converge. Physiol. Rev. 93, 107-135. doi: 10.1152/physrev.00016.2012

Gao, X., and Pan, D. (2001). TSC1 and TSC2 tumor suppressors antagonize insulin signaling in cell growth. Genes Dev. 15, 1383-1392. doi: 10.1101/gad.901101

Géminard, C., Rulifson, E. J., and Léopold, P. (2009). Remote control of insulin secretion by fat cells in Drosophila. Cell Metab. 10, 199-207. doi: 10.1016/j.cmet. 2009.08.002

Glossop, N. R., and Hardin, P. E. (2002). Central and peripheral circadian oscillator mechanisms in flies and mammals. J. Cell Sci. 115(Pt. 17), 3369-3377.

González, A., and Hall, M. N. (2017). Nutrient sensing and TOR signaling in yeast and mammals. EMBO J. 36, 397-408. doi: 10.15252/embj.201696010

Green, C. B., Takahashi, J. S., and Bass, J. (2008). The meter of metabolism. Cell 134, 728-742. doi: 10.1016/j.cell.2008.08.022

Grewal, S. S., Evans, J. R., and Edgar, B. A. (2007). Drosophila TIF-IA is required for ribosome synthesis and cell growth and is regulated by the TOR pathway. J. Cell Biol. 179, 1105-1113. doi: 10.1083/jcb.200709044

Guertin, D. A., Guntur, K. V., Bell, G. W., Thoreen, C. C., and Sabatini, D. M. (2006). Functional genomics identifies TOR-regulated genes that control growth and division. Curr. Biol. 16, 958-970. doi: 10.1016/j.cub.2006. 03.084

Hakes, A. E., and Brand, A. H. (2019). Neural stem cell dynamics: the development of brain tumours. Curr. Opin. Cell Biol. 60, 131-138. doi: 10.1016/j.ceb.2019.06. 001

Hara, R., Wan, K., Wakamatsu, H., Aida, R., Moriya, T., Akiyama, M., et al. (2001). Restricted feeding entrains liver clock without participation of the suprachiasmatic nucleus. Genes Cells 6, 269-278. doi: 10.1046/j.1365-2443. 2001.00419.x

Hardin, P. E., and Panda, S. (2013). Circadian timekeeping and output mechanisms in animals. Curr. Opin. Neurobiol. 23, 724-731. doi: 10.1016/j.conb.2013.02.018

Heisenberg, M. (2003). Mushroom body memoir: from maps to models. Nat. Rev. Neurosci. 4, 266-275. doi: 10.1038/nrn1074

Hermann-Luibl, C., and Helfrich-Förster, C. (2015). Clock network in Drosophila. Curr. Opin. Insect Sci. 7, 65-70.

Hernandez-Verdun, D. (2011). Assembly and disassembly of the nucleolus during the cell cycle. Nucleus 2, 189-194. doi: 10.4161/nucl.2.3.16246

Hietakangas, V., and Cohen, S. M. (2009). Regulation of tissue growth through nutrient sensing. Annu. Rev. Genet. 43, 389-410. doi: 10.1146/annurev-genet102108-134815

Homem, C. C., and Knoblich, J. A. (2012). Drosophila neuroblasts: a model for stem cell biology. Development 139, 4297-4310. doi: 10.1242/dev.080515

Homem, C. C., Repic, M., and Knoblich, J. A. (2015). Proliferation control in neural stem and progenitor cells. Nat. Rev. Neurosci. 16, 647-659. doi: 10.1038/ nrn4021

Hughes, M. E., Grant, G. R., Paquin, C., Qian, J., and Nitabach, M. N. (2012). Deep sequencing the circadian and diurnal transcriptome of Drosophila brain. Genome Res. 22, 1266-1281. doi: 10.1101/gr.128876.111 
Inoki, K., Li, Y., Zhu, T., Wu, J., and Guan, K. L. (2002). TSC2 is phosphorylated and inhibited by Akt and suppresses mTOR signalling. Nat. Cell Biol. 4, 648-657. doi: 10.1038/ncb839

Ito, K., and Hotta, Y. (1992). Proliferation pattern of postembryonic neuroblasts in the brain of Drosophila melanogaster. Dev. Biol. 149, 134-148. doi: 10.1016/ 0012-1606(92)90270-q

Jouffe, C., Cretenet, G., Symul, L., Martin, E., Atger, F., Naef, F., et al. (2013). The circadian clock coordinates ribosome biogenesis. PLoS Biol. 11:e1001455. doi: 10.1371/journal.pbio.1001455

Kaneko, M., Helfrich-Förster, C., and Hall, J. C. (1997). Spatial and temporal expression of the period and timeless genes in the developing nervous system of Drosophila: newly identified pacemaker candidates and novel features of clock gene product cycling. J. Neurosci. 17, 6745-6760. doi: 10.1523/JNEUROSCI.1717-06745.1997

Keene, A. C., Mazzoni, E. O., Zhen, J., Younger, M. A., Yamaguchi, S., Blau, J., et al. (2011). Distinct visual pathways mediate Drosophila larval light avoidance and circadian clock entrainment. J. Neurosci. 31, 6527-6534. doi: 10.1523/ JNEUROSCI.6165-10.2011

Khapre, R. V., Patel, S. A., Kondratova, A. A., Chaudhary, A., Velingkaar, N., Antoch, M. P., et al. (2014). Metabolic clock generates nutrient anticipation rhythms in mTOR signaling. Aging 6, 675-689. doi: 10.18632/aging.10 0686

Kim, E., Goraksha-Hicks, P., Li, L., Neufeld, T. P., and Guan, K. L. (2008). Regulation of TORC1 by Rag GTPases in nutrient response. Nat. Cell Biol. 10, 935-945. doi: 10.1038/ncb1753

Klarsfeld, A., Picot, M., Vias, C., Chélot, E., and Rouyer, F. (2011). Identifying specific light inputs for each subgroup of brain clock neurons in Drosophila larvae. J. Neurosci. 31, 17406-17415. doi: 10.1523/JNEUROSCI.5159-10.2011

Konopka, R. J., and Benzer, S. (1971). Clock mutants of Drosophila melanogaster. Proc. Natl. Acad. Sci. U.S.A. 68, 2112-2116. doi: 10.1073/pnas.68.9.2112

Koyama, T., Texada, M. J., Halberg, K. A., and Rewitz, K. (2020). Metabolism and growth adaptation to environmental conditions in Drosophila. Cell. Mol. Life Sci. 77, 4523-4551. doi: 10.1007/s00018-020-03547-2

Kraft, K. F., Massey, E. M., Kolb, D., Walldorf, U., and Urbach, R. (2016). Retinal homeobox promotes cell growth, proliferation and survival of mushroom body neuroblasts in the Drosophila brain. Mech. Dev. 142, 50-61. doi: 10.1016/j.mod. 2016.07.003

Kurusu, M., Maruyama, Y., Adachi, Y., Okabe, M., Suzuki, E., and FurukuboTokunaga, K. (2009). A conserved nuclear receptor, Tailless, is required for efficient proliferation and prolonged maintenance of mushroom body progenitors in the Drosophila brain. Dev. Biol. 326, 224-236. doi: 10.1016/j. ydbio.2008.11.013

Kurusu, M., Nagao, T., Walldorf, U., Flister, S., Gehring, W. J., and FurukuboTokunaga, K. (2000). Genetic control of development of the mushroom bodies, the associative learning centers in the Drosophila brain, by the eyeless, twin of eyeless, and Dachshund genes. Proc. Natl. Acad. Sci. U.S.A. 97, 2140-2144. doi: 10.1073/pnas.040564497

Lamia, K. A., Storch, K. F., and Weitz, C. J. (2008). Physiological significance of a peripheral tissue circadian clock. Proc. Natl. Acad. Sci. U.S.A. 105, 15172-15177. doi: $10.1073 /$ pnas.0806717105

Lanet, E., and Maurange, C. (2014). Building a brain under nutritional restriction: insights on sparing and plasticity from Drosophila studies. Front. Physiol. 5:117. doi: 10.3389/fphys.2014.00117

Liu, T., Mahesh, G., Houl, J. H., and Hardin, P. E. (2015). Circadian activators are expressed days before they initiate clock function in late pacemaker neurons from Drosophila. J. Neurosci. 35, 8662-8671. doi: 10.1523/JNEUROSCI.025015.2015

Malpel, S., Klarsfeld, A., and Rouyer, F. (2002). Larval optic nerve and adult extra-retinal photoreceptors sequentially associate with clock neurons during Drosophila brain development. Development 129, 1443-1453.

Marcheva, B., Ramsey, K. M., Buhr, E. D., Kobayashi, Y., Su, H., Ko, C. H., et al. (2010). Disruption of the clock components CLOCK and BMAL1 leads to hypoinsulinaemia and diabetes. Nature 466, 627-631. doi: 10.1038/ nature 09253

Maszewski, J., and Kwiatkowska, M. (1984). Number, size, and transcriptional activity of nucleoli during different periods of interphase in antheridial filaments of Chara vulgaris L. Folia Histochem. Cytobiol. 22, 9-19.
Maurange, C., Cheng, L., and Gould, A. P. (2008). Temporal transcription factors and their targets schedule the end of neural proliferation in Drosophila. Cell 133, 891-902. doi: 10.1016/j.cell.2008.03.034

Maury, E. (2019). Off the clock: from circadian disruption to metabolic disease. Int. J. Mol. Sci. 20:1597. doi: 10.3390/ijms20071597

Mazzoni, E. O., Desplan, C., and Blau, J. (2005). Circadian pacemaker neurons transmit and modulate visual information to control a rapid behavioral response. Neuron 45, 293-300. doi: 10.1016/j.neuron.2004.12.038

Mota, M. C., Silva, C. M., Balieiro, L., Fahmy, W. M., and Crispim, C. A. (2017). Social jetlag and metabolic control in non-communicable chronic diseases: a study addressing different obesity statuses. Sci. Rep. 7:6358. doi: 10.1038/ s41598-017-06723-w

Neumüller, R. A., Richter, C., Fischer, A., Novatchkova, M., Neumüller, K. G., and Knoblich, J. A. (2011). Genome-wide analysis of self-renewal in Drosophila neural stem cells by transgenic RNAi. Cell Stem Cell 8, 580-593. doi: 10.1016/j. stem.2011.02.022

Otsuki, L., and Brand, A. H. (2018). Cell cycle heterogeneity directs the timing of neural stem cell activation from quiescence. Science 360, 99-102. doi: 10.1126/ science.aan 8795

Panda, S., Hogenesch, J. B., and Kay, S. A. (2002). Circadian rhythms from flies to human. Nature 417, 329-335. doi: 10.1038/417329a

Patke, A., Young, M. W., and Axelrod, S. (2020). Molecular mechanisms and physiological importance of circadian rhythms. Nat. Rev. Mol. Cell Biol. 21, 67-84. doi: 10.1038/s41580-019-0179-2

Potter, C. J., Pedraza, L. G., and Xu, T. (2002). Akt regulates growth by directly phosphorylating Tsc2. Nat. Cell Biol. 4, 658-665. doi: 10.1038/ncb840

Russell, R. C., Fang, C., and Guan, K. L. (2011). An emerging role for TOR signaling in mammalian tissue and stem cell physiology. Development 138, 3343-3356. doi: $10.1242 /$ dev. 058230

Saxton, R. A., and Sabatini, D. M. (2017). mTOR Signaling in Growth. Metab. Dis. Cell 168, 960-976. doi: 10.1016/j.cell.2017.02.004

Sehgal, A., Price, J., and Young, M. W. (1992). Ontogeny of a biological clock in Drosophila melanogaster. Proc. Natl. Acad. Sci. U.S.A. 89, 1423-1427. doi: 10.1073/pnas.89.4.1423

Shi, M., and Zheng, X. (2013). Interactions between the circadian clock and metabolism: there are good times and bad times. Acta Biochim. Biophys. Sin (Shanghai) 45, 61-69. doi: 10.1093/abbs/gms110

Shim, J., Gururaja-Rao, S., and Banerjee, U. (2013). Nutritional regulation of stem and progenitor cells in Drosophila. Development 140, 4647-4656. doi: 10.1242/ dev.079087

Siegrist, S. E., Haque, N. S., Chen, C. H., Hay, B. A., and Hariharan, I. K. (2010). Inactivation of both Foxo and reaper promotes long-term adult neurogenesis in Drosophila. Curr. Biol. 20, 643-648. doi: 10.1016/j.cub.2010. 01.060

Sousa-Nunes, R., Yee, L. L., and Gould, A. P. (2011). Fat cells reactivate quiescent neuroblasts via TOR and glial insulin relays in Drosophila. Nature 471, 508-512. doi: 10.1038/nature09867

Spéder, P., and Brand, A. H. (2014). Gap junction proteins in the blood-brain barrier control nutrient-dependent reactivation of Drosophila neural stem cells. Dev. Cell 30, 309-321. doi: 10.1016/j.devcel.2014.05.021

Stępiński, D. (2018). The nucleolus, an ally, and an enemy of cancer cells. Histochem. Cell Biol. 150, 607-629. doi: 10.1007/s00418-018-1706-5

Truman, J. W., and Bate, M. (1988). Spatial and temporal patterns of neurogenesis in the central nervous system of Drosophila melanogaster. Dev. Biol. 125, 145-157. doi: 10.1016/0012-1606(88)90067-x

Walsh, K. T., and Doe, C. Q. (2017). Drosophila embryonic type II neuroblasts: origin, temporal patterning, and contribution to the adult central complex. Development 144, 4552-4562. doi: 10.1242/dev.157826

Wijnen, H., Naef, F., Boothroyd, C., Claridge-Chang, A., and Young, M. W. (2006). Control of daily transcript oscillations in Drosophila by light and the circadian clock. PLoS Genet. 2:e39. doi: 10.1371/journal.pgen.0020039

Xu, K., DiAngelo, J. R., Hughes, M. E., Hogenesch, J. B., and Sehgal, A. (2011). The circadian clock interacts with metabolic physiology to influence reproductive fitness. Cell Metab. 13, 639-654. doi: 10.1016/j.cmet.2011.05.001

Xu, K., Zheng, X., and Sehgal, A. (2008). Regulation of feeding and metabolism by neuronal and peripheral clocks in Drosophila. Cell Metab. 8, 289-300. doi: 10.1016/j.cmet.2008.09.006 
Yasugi, T., and Nishimura, T. (2016). Temporal regulation of the generation of neuronal diversity in Drosophila. Dev. Growth Differ. 58, 73-87. doi: 10.1111/ dgd. 12245

Zhang, H., Stallock, J. P., Ng, J. C., Reinhard, C., and Neufeld, T. P. (2000). Regulation of cellular growth by the Drosophila target of rapamycin dTOR. Genes Dev. 14, 2712-2724. doi: 10.1101/gad.835000

Zhao, J., Warman, G. R., Stanewsky, R., and Cheeseman, J. F. (2019). Development of the molecular circadian clock and its light sensitivity in drosophila melanogaster. J. Biol. Rhythms 34, 272-282. doi: 10.1177/0748730419836818

Zheng, X., and Sehgal, A. (2010). AKT and TOR signaling set the pace of the circadian pacemaker. Curr. Biol. 20, 1203-1208. doi: 10.1016/j.cub.2010.05.027

Zhu, C. C., Boone, J. Q., Jensen, P. A., Hanna, S., Podemski, L., Locke, J., et al. (2008). Drosophila activin- and the activin-like product dawdle function redundantly to regulate proliferation in the larval brain. Development 135 , 513-521. doi: 10.1242/dev.010876
Zordan, M. A., and Sandrelli, F. (2015). Circadian clock dysfunction and psychiatric disease: could fruit flies have a say? Front. Neurol. 6:80. doi: 10.3389/ fneur.2015.00080

Conflict of Interest: The authors declare that the research was conducted in the absence of any commercial or financial relationships that could be construed as a potential conflict of interest.

Copyright (C) 2021 Dapergola, Menegazzi, Raabe and Hovhanyan. This is an openaccess article distributed under the terms of the Creative Commons Attribution License (CC BY). The use, distribution or reproduction in other forums is permitted, provided the original author(s) and the copyright owner(s) are credited and that the original publication in this journal is cited, in accordance with accepted academic practice. No use, distribution or reproduction is permitted which does not comply with these terms. 